DOI: $10.21554 / \mathrm{hrr} .041915$

\title{
ISOSCELES TRIANGLES ON THE SIDES OF A TRIANGLE
}

Sead Rešić ${ }^{1}$

Alma Šehanović Amila Osmić

Department of Mathematics, Faculty of Science, University of Tuzla, Bosnia and Herzegovina Gymnasium Mesa Selimovic Tuzla, Bosnia and Herzegovina

Construction and Geodesy School of Tuzla, Bosnia and Herzegovina
Original scientific paper

Received: 2019/2/22

Accepted: 2019/4/4

\begin{abstract}
Famous construction of Fermat-Toricelly point of a triangle leads to the question is there a similar way to construct other isogonic centers of a triangle in a similar way. For a purpose we remember that Fermat-Torricelli point of a triangle $\triangle A B C$ is obtained by constructing equilateral triangles outwardly on the sides $A B, B C$ and $C A$. If we denote thirth vertices of those triangles by $C_{1}, A_{1}$ and $B_{1}$ respectively, then the lines $A A_{1}, B B_{1}$ and $C C_{1}$ concurr at the Fermat-Torricelli point of a triangle $\triangle A B C$ (Van Lamoen, 2003). In this work we present the condition for the concurrence, of the lines $A A_{l}, B B_{1}$ and $C_{l}$, where $C_{l}, A_{1}$ and $B_{1}$ are the vertices of an isosceles triangles constructed on the sides $A B, B C$ and $C A$ (not necessarily outwordly) of a triangle $\triangle A B C$. The angles at this work are strictly positive directed so we recommend the reader to pay attention to this fact.
\end{abstract}

Keywords: Ceva, Menelaus, Stewartes, cevian, concurrency, collinearity, Fermat, Torricelly

\section{INTRODUCTION}

Leading idea for this work was Napoleon Triangles and Kiepert Perspectors, submitted by Floor van Lamoen (2003) to Forum Geometricorum in which the complex numbers are used to show the existance and the construction of Fermat-Toricelly point. Observing the hystorical facts we can se the Fermats-Toricelly point is one of the extremal points of a triangle, same as the centroid is. Namely if the point $\mathrm{O}$ is constructed in the plane of a triangle $\triangle \mathrm{ABC}$ then the sum $\mathrm{AO}+\mathrm{BO}+\mathrm{CO}$ is minimal if and onl if $\mathrm{O}$ coinsides with FermatToricellis point of a triangle $\triangle \mathrm{ABC}$ (Prasolov, 2001). Later as a special case we will see this one leads to the condition $\measuredangle A O C=\Varangle B O A=\Varangle C O B=\frac{2 \pi}{3}$.

The sum $\mathrm{AO}^{2}+\mathrm{BO}^{2}+\mathrm{CO}^{2}$ is minimal if and only if $\mathrm{O}$ coinsides with the centroid of a triangle $\triangle \mathrm{ABC}$ (Altshiller-Court, 2007). One can ask the quaestion when the sum $\mathrm{AO}^{3}+\mathrm{BO}^{3}+\mathrm{CO}^{3}$ is minimal, or some other questions. The theorem we present shows that any point in the plane of a triangle can be constructed using an issoceles triangles and certain condition.

\footnotetext{
${ }^{1}$ Correspondence to:

Sead Rešić, Department of Mathematics, Faculty of Science, University of Tuzla, Bosnia and Herzegovina

Univerzitetska 4, 75000 Tuzla, Bosnia and Herzegovina

Phone:+38761 101230

E-mail: sresic@hotmail.com
} 
MAIN THEOREM

and $\mathrm{C}_{1}$ lie in the plane of a triangle such that $\measuredangle \mathrm{ACB}^{1}=$ $\Varangle \mathrm{B}_{1} \mathrm{AC}=\varphi \quad \Varangle \mathrm{BAC}_{1}=\Varangle \mathrm{C}_{1} \mathrm{BA}=\omega$ and $\measuredangle \mathrm{CBA}_{1}=\Varangle \mathrm{A}_{1}$

Theorem 1. In a nondegenerated triangle $\measuredangle \mathrm{ABC}, \measuredangle \quad \mathrm{CB}=\delta$. The lines $\mathrm{AA}_{1}, \mathrm{BB}_{1}$ and $\mathrm{CC}_{1}$ are concurrent or $\mathrm{CAB}=\alpha, \quad \measuredangle \mathrm{ABC}=\beta, \quad \measuredangle \mathrm{BCA}=\gamma$. Let the points $\mathrm{A}_{1}, \mathrm{~B}_{1} \quad$ parallel if and only if

$$
\begin{aligned}
& \sin (\omega+\alpha) \cdot \sin (\varphi+\gamma) \cdot \sin (\beta+\delta)=\sin (\omega+\beta) \cdot \sin (\varphi+\alpha) \cdot(\delta+\gamma) \Leftrightarrow \\
& \sin (\varphi-\omega) \cdot \cos (2 \alpha-\delta)+\sin (\omega-\delta) \cdot \cos (2 \beta-\varphi)+\sin (\delta-\varphi) \cdot \cos (2 \gamma-\omega)=0
\end{aligned}
$$

\section{Proof:}

Let us consider the case

$$
\sin (\omega+\alpha) \cdot \sin (\varphi+\gamma) \cdot \sin (\beta+\delta) \cdot \sin (\omega+\beta) \cdot \sin (\varphi+\alpha) \cdot(\delta+\gamma)=0
$$

Let $\sin (\omega+\alpha)=0$. Since a triangle $\triangle A B C$ is nondegenerated, thus $\omega+\alpha \neq 0$ so we have $\omega+\alpha \in\{\pi, 2 \pi\}$.

Let $\omega+\alpha=\pi$, then $\measuredangle \mathrm{BAC}_{1}+\measuredangle \mathrm{CAB}=\pi$, which means Let $\mathrm{AA}_{1}$ and $\mathrm{CC}_{1}$ meet at $\mathrm{A}$ then $\mathrm{BB}_{1}$ also contains the $\mathrm{C}_{1}$ lies on the extension of the line $\mathrm{CA}$ such that $\mathrm{A}$ point $\mathrm{A}$. Thus $\mathrm{B}_{1}$ lies on the line $\mathrm{AB}$. Since $\measuredangle A C \mathrm{~B}_{1}=\measuredangle$ is between the points $\mathrm{C}$ and $\mathrm{C}_{1}$. Since $\measuredangle \mathrm{BAC}_{1}=\measuredangle \mathrm{C}_{1} \quad \mathrm{~B}_{1} \mathrm{AC}$, and $\alpha>\frac{\pi}{2}$, then $\mathrm{A}$ is between the points $\mathrm{B}$ and $\mathrm{BA}=\omega=\pi-\alpha$ then we have $2(\pi-\alpha)<\pi \Rightarrow \alpha>\frac{\pi}{2} . \quad \mathrm{B}_{1}$. Now we have $\measuredangle \mathrm{ACB}_{1}=\measuredangle \mathrm{B}_{1} \mathrm{AC}=\varphi=\omega$ so we have

$$
\varphi+\alpha=\pi \Rightarrow \sin (\varphi+\alpha)=0 \Rightarrow
$$

$$
\sin (\omega+\alpha) \cdot \sin (\varphi+\gamma) \cdot \sin (\beta+\delta)=0=\sin (\omega+\beta) \cdot \sin (\varphi+\alpha) \cdot(\delta+\gamma)
$$

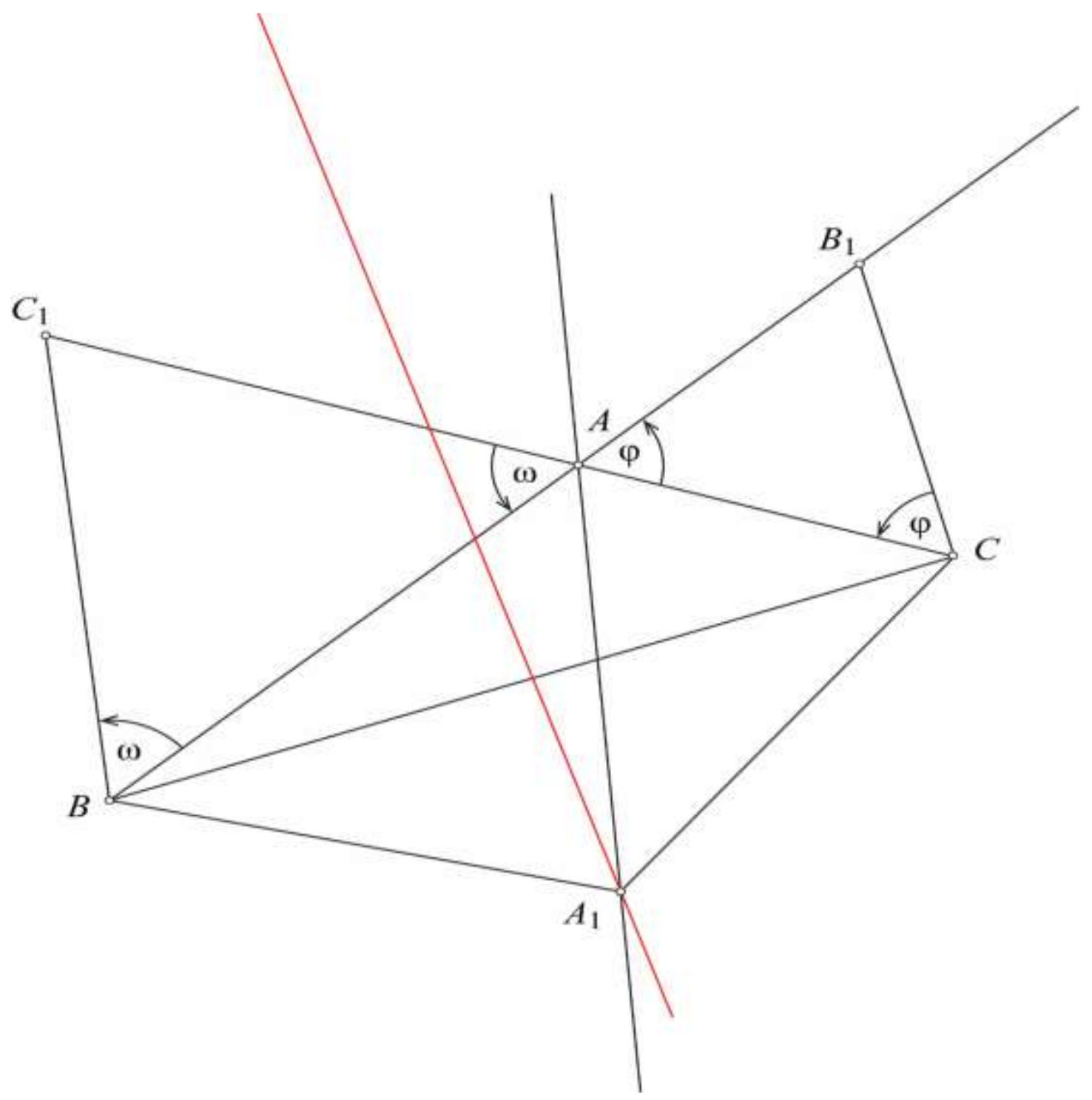

Figure 1. 
Then $\mathrm{A}_{1}$ is any point on the bisector of the segment $\mathrm{BC}$. point of a line through $\mathrm{B}$ parallel to $\mathrm{CA}$ and the bisector Let $\mathrm{AA}_{1}$ be parallel to $\mathrm{CC}_{1}$, then $\mathrm{A}_{1}$ lies on the line $\mathrm{CA}$. of the segment $\mathrm{CA}$.

If $\mathrm{BB}_{1}$ is also parallel to $\mathrm{CC}_{1}$ then $\mathrm{B}_{1}$ is an intersection

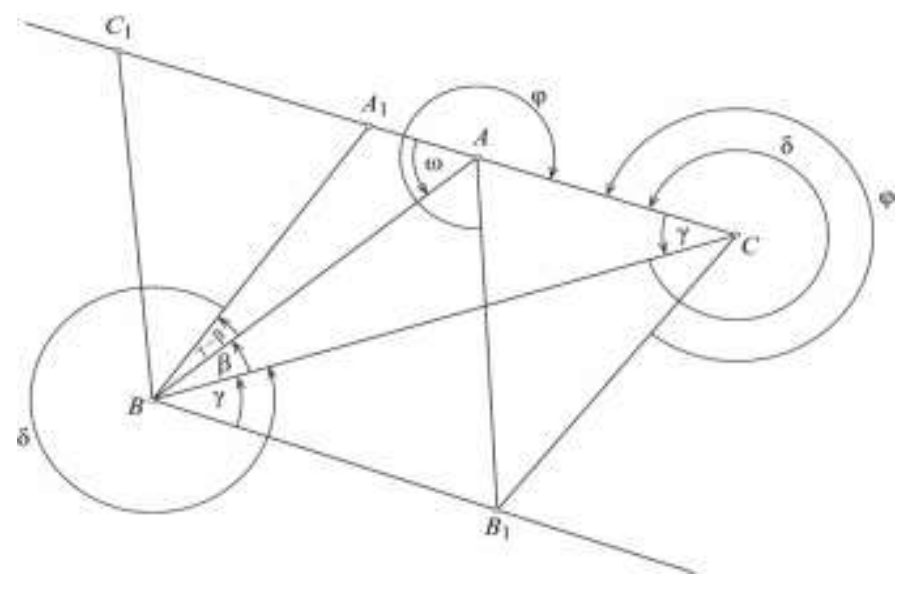

Figure 2.

But then we have

$$
\begin{gathered}
\Varangle A_{1} C B+\Varangle B C A=2 \pi \Rightarrow \delta+\gamma=2 \pi \Rightarrow \sin (\delta+\gamma)=0 \Rightarrow \\
\sin (\omega+\alpha) \cdot \sin (\varphi+\gamma) \cdot \sin (\beta+\delta)=0=\sin (\omega+\beta) \cdot \sin (\varphi+\alpha) \cdot(\delta+\gamma)
\end{gathered}
$$

Let $\omega+\alpha=2 \pi$ so the point $C_{1}$ is on the line CA such But then we have that $\mathrm{C}$ and $\mathrm{C}_{1}$ are on the same side of the point $\mathrm{A}$.

$$
\measuredangle B A C_{1}=\measuredangle C_{1} B A \Rightarrow 2 \pi-\measuredangle B A C_{1}=2 \pi-\measuredangle C_{1} B A \Rightarrow
$$

$\Varangle C_{1} A B=\Varangle A B C_{1} \Rightarrow \alpha<\frac{\pi}{2}$. Let $C C_{1}$ and $A A_{1}$ meet at the point $A$. Then $B B_{1}$ contains the point $A$ only if $B_{1}$ is on the line $B A$. Then we have

$$
\begin{gathered}
\measuredangle B_{1} A C=\measuredangle B A C_{1} \Rightarrow \varphi=\omega \Rightarrow \varphi+\alpha=2 \pi \Rightarrow \sin (\varphi+\alpha)=0 \Rightarrow \\
\sin (\omega+\alpha) \cdot \sin (\varphi+\gamma) \cdot \sin (\beta+\delta)=0=\sin (\omega+\beta) \cdot \sin (\varphi+\alpha) \cdot(\delta+\gamma)
\end{gathered}
$$

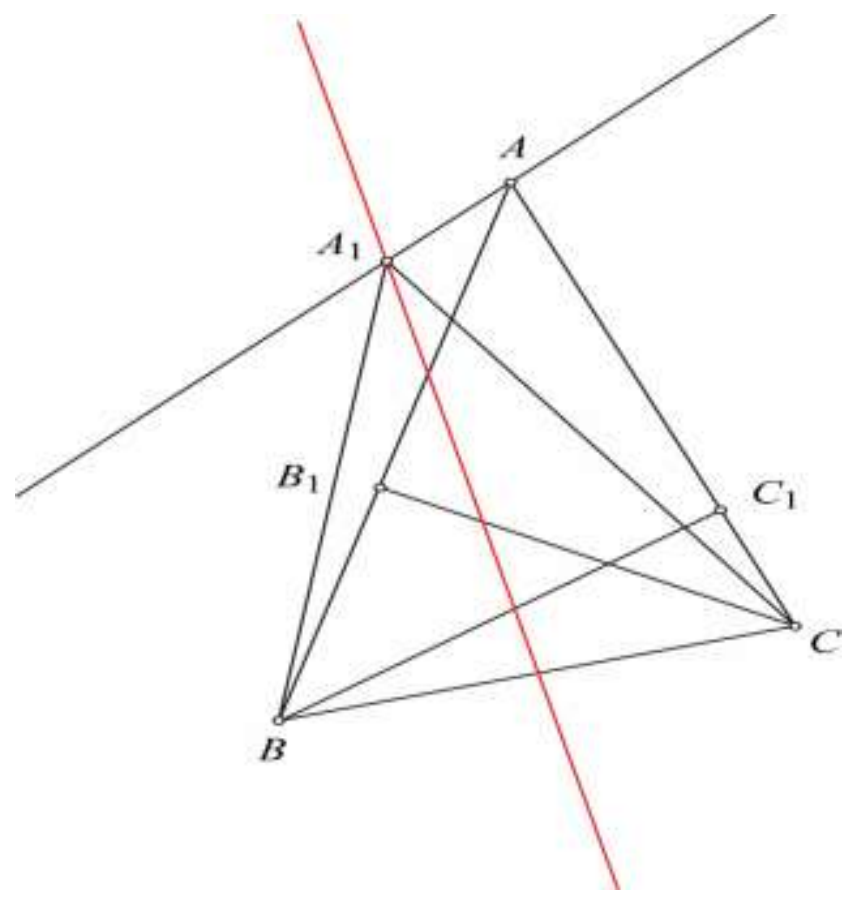

Figure 3. 
Let $\mathrm{AA}_{1}$ be parallel to the line $\mathrm{CC}_{1}$, Then $\mathrm{BB}_{1}$ paral- throught $\mathrm{B}$ parallel to $\mathrm{AC}$ and the bisector of the seglel to them so $\mathrm{B}_{1}$ is an interesection point of the line ment AC. But then

$$
\Varangle A_{1} C B+\Varangle B C A=2 \pi \Rightarrow \delta+\gamma=2 \pi \Rightarrow \sin (\delta+\gamma)=0 \Rightarrow
$$

$$
\sin (\omega+\alpha) \cdot \sin (\varphi+\gamma) \cdot \sin (\beta+\delta)=0=\sin (\omega+\beta) \cdot \sin (\varphi+\alpha) \cdot(\delta+\gamma)
$$

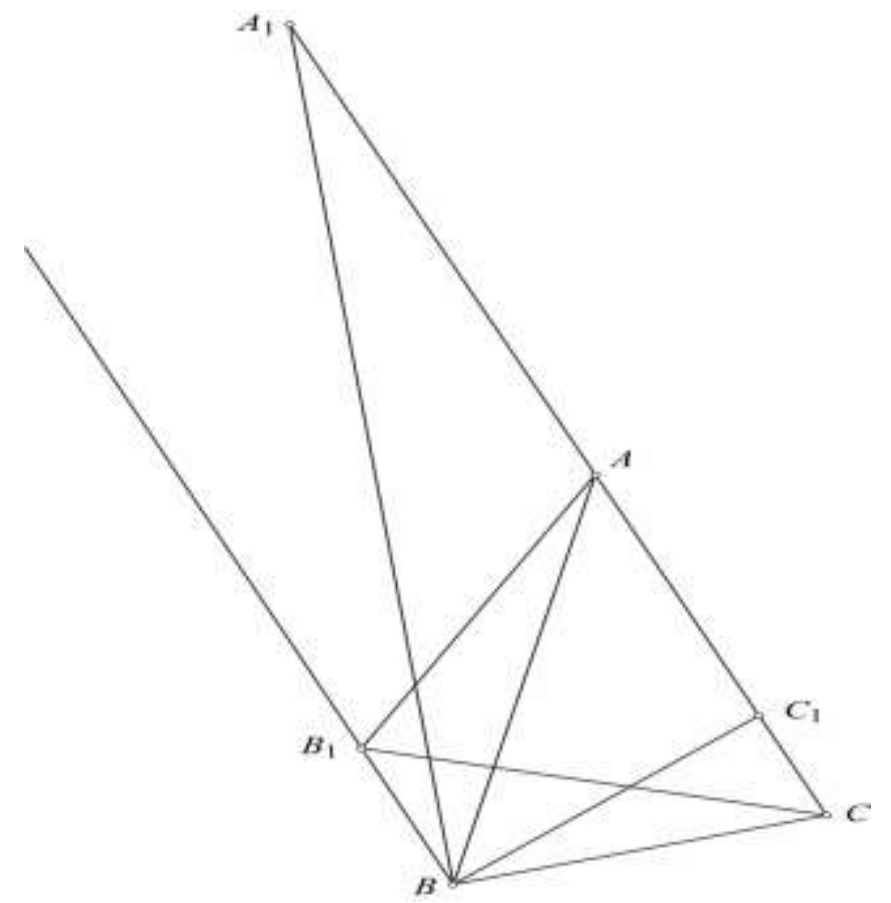

Figure 4.

Similarly we reconsider the cases remained from the equation

$$
\sin (\omega+\alpha) \cdot \sin (\varphi+\gamma) \cdot \sin (\beta+\delta) \cdot \sin (\omega+\beta) \cdot \sin (\varphi+\alpha) \cdot(\delta+\gamma)=0
$$

As we can notice, the intersection points of the lines or the point at infinity.

$\mathrm{AA}_{1}, \mathrm{BB}_{1}$ and $\mathrm{CC}_{1}$ are the triangle vertices $\mathrm{A}, \mathrm{B}$ and $\mathrm{C}$ Suppose now that

$$
\sin (\omega+\alpha) \cdot \sin (\varphi+\gamma) \cdot \sin (\beta+\delta) \cdot \sin (\omega+\beta) \cdot \sin (\varphi+\alpha) \cdot(\delta+\gamma) \neq 0
$$

Consider the points $\mathrm{A}$ and $\mathrm{A}_{1}$ being from distinct to $\mathrm{BC}$ meets lines $\mathrm{AB}$ and $\mathrm{AC}$ at the points $\mathrm{D}$ and $\mathrm{E}$ sides of a line $\mathrm{BC}$. Let the line $\mathrm{AA}_{1}$ meets the line respectively. From the similarity $\triangle \mathrm{ABC} \sim \triangle \mathrm{ADE}$ we $\mathrm{BC}$ at the point $\mathrm{A}^{\prime}$. Let the line through $\mathrm{A}_{1}$ parallel have

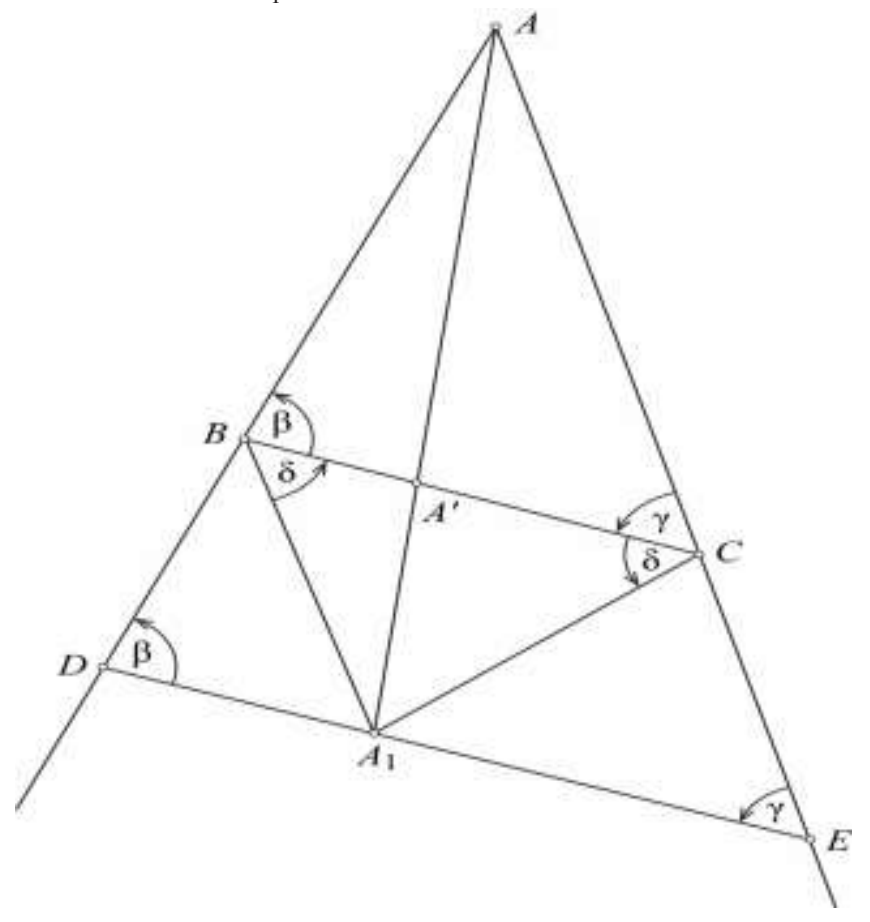

Figure 5. 
$\frac{A_{1} D}{A_{1} E}=\frac{B A^{\prime}}{A^{\prime} C}$

From the sine theorem we have

$A_{1} D=\frac{A_{1} B}{\sin \beta} \cdot \sin (\beta+\delta)$

$A_{1} E=\frac{A_{1} C}{\sin \gamma} \cdot \sin (\gamma+\delta)$

Dividing we get

$\frac{B A^{\prime}}{A^{\prime} C}=\frac{\sin \gamma}{\sin \beta} \cdot \frac{\sin (\beta+\delta)}{\sin (\gamma+\delta)}$
Let now $A$ and $A_{1}$ be from the same side of the line $\mathrm{BC}$ Let the line $\mathrm{AA}_{1}$ meets the line $\mathrm{BC}$ at the point $\mathrm{A}^{\prime}$. Let the line through $\mathrm{A}_{1}$ parallel to $\mathrm{BC}$ meets lines $\mathrm{AB}$ and $\mathrm{AC}$ at the points $\mathrm{D}$ and $\mathrm{E}$ respectively. From the similarity $\triangle \mathrm{ABC} \sim \triangle \mathrm{ADE}$ we have

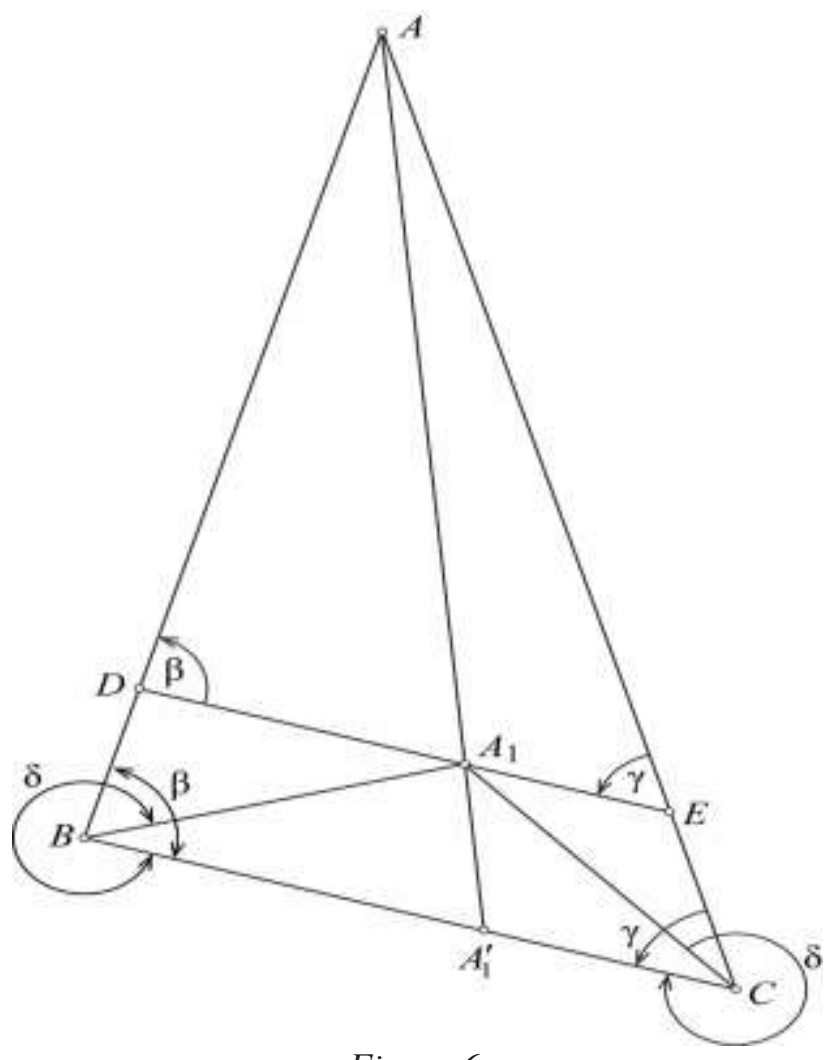

Figure 6.

$\frac{A_{1} D}{A_{1} E}=\frac{B A^{\prime}}{A^{\prime} C}$

From the sine theorem we have

$A_{1} D=\frac{A_{1} B}{\sin \beta} \cdot \sin (\beta+\delta-2 \pi)$

$A_{1} E=\frac{A_{1} C}{\sin \gamma} \cdot \sin (\gamma+\delta-2 \pi)$
Dividing we get

$\frac{B A^{\prime}}{A^{\prime} C}=\frac{\sin \gamma}{\sin \beta} \cdot \frac{\sin (\beta+\delta)}{\sin (\gamma+\delta)}$

So in any case we have

$\frac{B A^{\prime}}{A^{\prime} C}=\frac{\sin \gamma}{\sin \beta} \cdot \frac{\sin (\beta+\delta)}{\sin (\gamma+\delta)}$ 
Let us define the points $\mathrm{B}^{\prime}$ and $\mathrm{C}^{\prime}$ similarly. $\mathrm{By}$ and $\mathrm{CC}_{1}$ meet at the point or are parallel if and Cevas theorem (Kedlaya, 1999) the lines $\mathrm{AA}_{1}, \mathrm{BB}_{1}$ only if

$$
\begin{gathered}
\frac{B A^{\prime}}{A^{\prime} C} \cdot \frac{C B^{\prime}}{B^{\prime} A} \cdot \frac{A C^{\prime}}{C^{\prime} B}=1 \Leftrightarrow \\
\frac{\sin (\beta+\delta) \cdot \sin (\varphi+\gamma) \cdot \sin (\omega+\alpha)}{\sin (\gamma+\delta) \cdot \sin (\varphi+\alpha) \cdot \sin (\omega+\beta)}=1 \Leftrightarrow \\
\sin (\varphi-\omega) \cdot \cos (2 \alpha-\delta)+\sin (\omega-\delta) \cdot \cos (2 \beta-\varphi)+\sin (\delta-\varphi) \cdot \cos (2 \gamma-\omega)=0
\end{gathered}
$$

\section{CONSEQUENCES WHEN $\delta=\varphi=\omega$}

Corollary 1. On the sides of a nondegenerated triangle $\triangle \mathrm{ABC}$ are constructed regular n-gons outwardly, $\mathrm{AC}_{2} \ldots$ $\mathrm{C}_{\mathrm{n}-1} \mathrm{~B}, \mathrm{BA}_{2} \ldots \mathrm{A}_{\mathrm{n}-1} \mathrm{C}$ and $\mathrm{CB}_{2} \ldots \mathrm{B}_{\mathrm{n}-1} \mathrm{~A}$.Let $\mathrm{C}_{1}, \mathrm{~A}_{1}$ and $\mathrm{B}_{1}$ be the centers of those polygones respectively. Then the lines $\mathrm{AA}_{1}, \mathrm{BB}_{1}$ and $\mathrm{CC}_{1}$ concurr.

\section{Proof:}

Since the triangles $\triangle \mathrm{AC}_{1} \mathrm{~B}, \Delta \mathrm{BA}_{1} \mathrm{C}$ and $\triangle \mathrm{CB}_{1} \mathrm{~A}$ are an issoceles triangles constructed on the sides of nondegenerated triangle $\triangle \mathrm{ABC}$ and $\delta=\varphi=\omega=\frac{n-2}{2 n} \pi$, applying the theorem 1 in its second equivalent form directly implies the claim.

Corollary 2. On the sides of a nondegenerated triangle $\triangle \mathrm{ABC}$ are constructed regular $2 \mathrm{n}+1$-gons outwardly, $\mathrm{AC}_{2} \ldots \mathrm{C}_{2 \mathrm{n}} \mathrm{B}, \mathrm{BA}_{2} \ldots \mathrm{A}_{2 \mathrm{n}} \mathrm{C}$ and $\mathrm{CB}_{2} \ldots \mathrm{B}_{2 \mathrm{n}} \mathrm{A}$.. Then the lines $\mathrm{AA}_{\mathrm{n}+1}, \mathrm{BB}_{\mathrm{n}+1}$ and $\mathrm{CC}_{\mathrm{n}+1}$ concurr.

\section{Proof:}

Since the triangles $\Delta \mathrm{AC}_{\mathrm{n}+1} \mathrm{~B}, \quad \Delta \mathrm{BA}_{\mathrm{n}+1} \mathrm{C}$ and
$\Delta \mathrm{CB}_{\mathrm{n}+1} \mathrm{~A}$ are an issoceles triangles constructed on the sides of nondegenerated triangle $\triangle \mathrm{ABC}$ and $\delta=\varphi=\omega=\frac{n-1}{2 n} \pi$ applying the theorem 1 in its second equivalent form directly implies the claim.

Corollary 3. On the sides of a nondegenerated triangle $\triangle \mathrm{ABC}$ are constructed regular 2n-gons outwardly, $\mathrm{AC}_{2} \ldots \mathrm{C}_{2 \mathrm{n}-1} \mathrm{~B}, \mathrm{BA}_{2} \ldots \mathrm{A}_{2 \mathrm{n}-1} \mathrm{C}$ and $\mathrm{CB}_{2} \ldots \mathrm{B}_{2 \mathrm{n}-1}$. Let $\mathrm{C}_{1}, \mathrm{~A}_{1}$ and $B_{1}$ be themidpoints of the sides $A_{n} A_{n+1}, B_{n} B_{n+1}$ and $\mathrm{C}_{\mathrm{n}} \mathrm{C}_{\mathrm{n}+1}$ respectively. Then the lines $\mathrm{AA}_{1}, \mathrm{BB}_{1}$ and $\mathrm{CC}_{1}$ concurr.

\section{Proof:}

Since the triangles $\Delta \mathrm{AC}_{1} \mathrm{~B}, \Delta \mathrm{BA}_{1} \mathrm{C}$ and $\Delta \mathrm{CB}_{1} \mathrm{~A}$ are an issoceles triangles constructed on the sides of nondegenerated triangle $\triangle \mathrm{ABC}$ and $\delta=\varphi=\omega$, applying the theorem 1 in its second equivalent form directly implies the claim. The corollaries obviously hold when the polygons are constructed inwardly.

Let us just draw the case when all the triangles are outwards

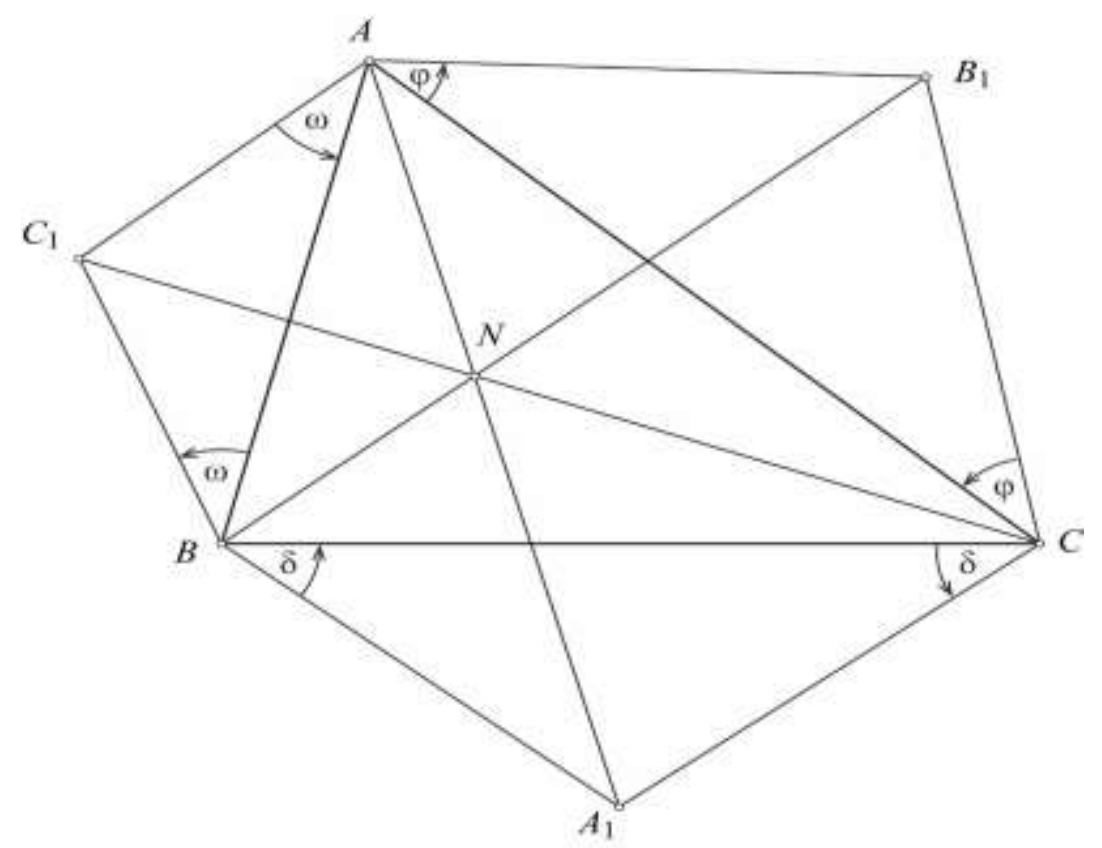

Figure 7. 


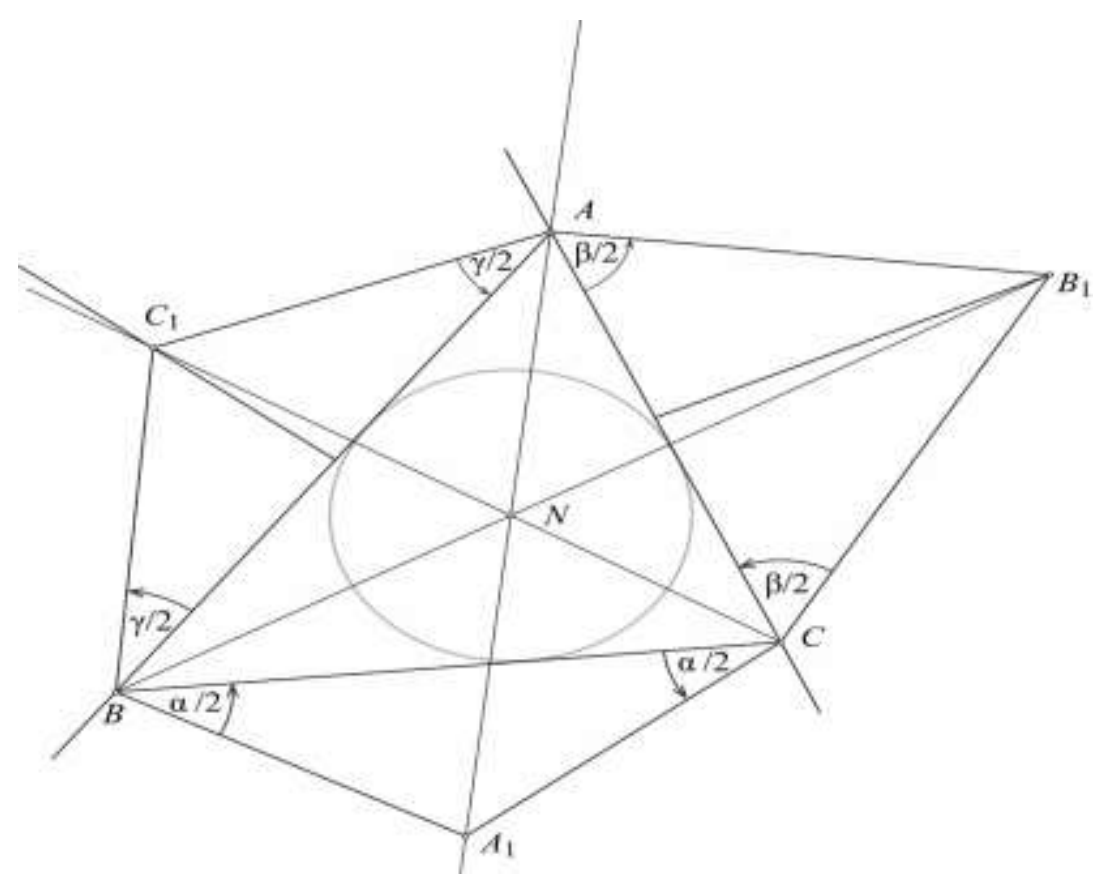

Figure 8.

This is the special case when the lies meet at the in- Then below is the special case when the lines meet at center.

Fermat-Toricelly point (Prasolov, 2001)

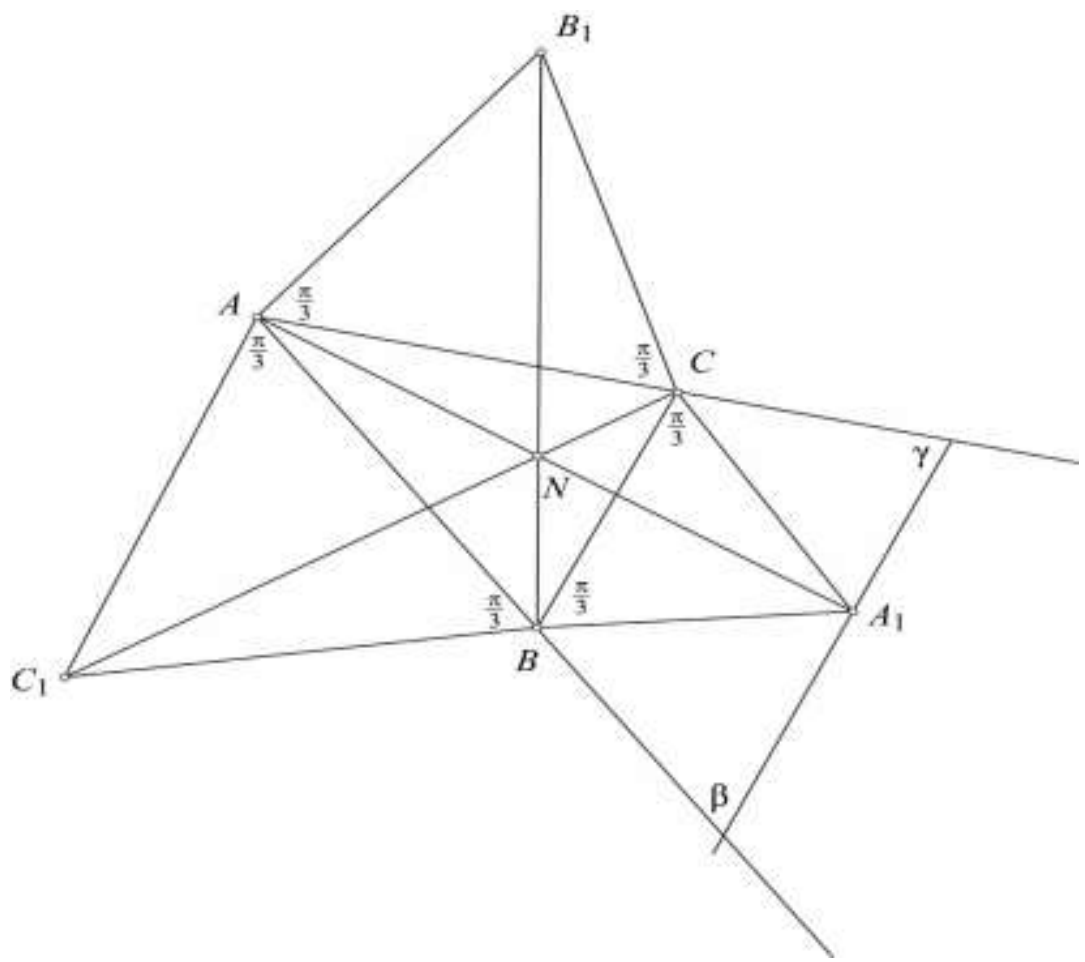

Figure 9.

\section{CONCLUSION}

Any point in the plane of nondegenerated triangle can be constructed using this method except the points belonging to the altitudes of the triangle excluding its vertices which can be constructed. This fact is obvious, any point can be connected to the vertices of a triangle, thus forming a line. The intersections of those three lines with the bisectors of the sides op- posing to the vertices respectively, form three vertices of required issoceles triangles, which is not the case only if the one of the points lie on the line containing the altitude. Then connecting this point to the vertex form a line parallel to the bisector of the opposing side, hence these two lines dont meet. So there is no required issoceles triangle. Also we can see that if the point is constructible this way, then the way of construction is unique. 


\section{REFERENCES}

Altshiller-Court, N. (2007). College Geometry: An Introduction to the Modern Geometry of the Triangle and the Circle. New York: Dover Publications, Inc. Mineola.

Kedlaya, K. (1999). Notes on Euclidean Geometry. Retrieved from http://web.math.rochester.edu/people/faculty/dangeba/geom-080399.pdf.
Prasolov, V. (2001). Problems in plane and solid geometry. (Vol. 1. Plane Geometry). translated and edited by Dimitry Leites. Retrieved from http://e.math.hr/old/afine/planegeo.pdf.

Van Lamoen, F. (2003). Napoleon Triangles and Kiepert Perspectors. Forum Geometricorum 3, pp. 65-71. 